\title{
Classic Spotlight, 1994 and 1995: Articles of Significant Interest Selected from the Journal of Virology Archives by the Editors
}

\begin{abstract}
ournal of Virology (JVI) marks its 50th year of publishing in 2017. To highlight particularly noteworthy $\mathrm{JVI}$ articles from over the years, 2017 issues are featuring Classic Spotlights selected from the archives by the editors. These Classic Spotlights are appearing chronologically, and in this issue, we have selected articles from 1994 and 1995.
\end{abstract}

Epstein-Barr Virus Latency in Blood Mononuclear Cells: Analysis of Viral Gene Transcription during Primary Infection and in the Carrier State

Epstein-Barr virus (EBV) is a gammaherpesvirus that is widespread in human populations. EBV infects two major cell types: pharyngeal epithelial cells, which appear to be susceptible to virus replication leading to release of infectious virions, and mature $B$ lymphocytes, which harbor the virus as a nonproductive (latent) infection. It had been shown previously that EBV can display different types of latent infection in B cells in vitro. Tierney et al. (R. J. Tierney, N. Steven, L. S. Young, and A. B. Rickinson, J Virol 68:7374-7385, 1994, http://jvi.asm.org/content/68/11/7374.abstract) addressed the question of the types of infection established by EBV in vivo by analyzing viral RNAs present in mononuclear cells freshly isolated from the blood of 14 infectious mononucleosis patients undergoing primary EBV infection and 6 long-term virus carriers. Reverse transcription-PCR amplifications were conducted using a panel of oligonucleotide probes that specifically detected (i) EBER1 RNA common to all forms of latency, (ii) transcripts from all six nuclear antigens (EBNA1, $-2,-3 A,-3 B,-3 C$, - LP), (iii) the latent membrane protein (LMP1 and -2A) mRNAs, and (iv) BZLF1 mRNA, a marker of the lytic cycle. The results showed that primary infection is associated with a transient virusdriven expansion of infected $B$ cells through a program of virus gene expression similar to that seen in in vitro-transformed cells and that long-term virus infection is associated with a switch to a more restricted form of latent protein expression. It has now been established that there are at least three types of EBV latency programs, which differ in the number of viral genes expressed. In type III latency, all 9 EBV latency proteins are expressed, whereas type II is more restricted; subsequently, EBV infection in B cells switches to a stringent form of viral latency, type I, in which only the EBNA1 protein is expressed in addition to noncoding viral RNAs. The more restricted form of latent protein expression may render the latently infected cells less susceptible to recognition by virus-specific cytotoxic T cells.

\section{CD4 ${ }^{+} \mathrm{T}$ Cells Are Required To Sustain CD8 ${ }^{+}$Cytotoxic T-Cell Responses during Chronic Viral Infection}

Matloubian et al. (M. Matloubian, R. J. Concepcion, and R. Ahmed, J Virol 68:80568063, 1994, http://jvi.asm.org/content/68/12/8056.abstract) examined the relative contributions of $\mathrm{CD}^{+}$and $\mathrm{CD}^{+}$T cells in controlling an acute or chronic lymphocytic choriomeningitis virus (LCMV) infection. Previous studies had shown that CD8 ${ }^{+}$cytotoxic T lymphocytes (CTLS) are essential for resolving an acute LCMV infection. However, $\mathrm{CD}^{+}{ }^{+} \mathrm{T}$ cells were not necessary, in that $\mathrm{CD} 4$-depleted mice were able to effectively control an acute LCMV infection. To analyze chronic infection, a panel of
Citation American Society for Microbiology. 2017. Classic Spotlight, 1994 and 1995: Articles of significant interest selected from the Journal of Virology archives by the editors. J Virol 91:e00671-17. https://doi.org/10.1128/JVI .00671-17.

Copyright $\odot 2017$ American Society for Microbiology. All Rights Reserved. 
lymphocyte-tropic and macrophage-tropic variants of LCMV that persist in adult mice for several months was used. In contrast to results seen with acute infection, adult mice given a single injection of a monoclonal antibody against CD4 at the time of virus challenge became lifelong carriers, and LCMV-specific CTL responses were lost in chronically infected CD4-depleted mice. These results show that $\mathrm{CD}^{+}{ }^{+} \mathrm{T}$ cells are dispensable for short-term acute infection in which $C D 8^{+}$CTL activity does not need to be sustained for more than 2 weeks. However, under conditions of chronic infection, in which $\mathrm{CD}^{+}$CTLs take several months to clear the infection, CD4 ${ }^{+}$T-cell function is critical, indicating that $\mathrm{CD} 4^{+} \mathrm{T}$ cells play an important role in sustaining virus-specific $\mathrm{CD}^{+}$CTL during chronic LCMV infection.

Lower In Vivo Mutation Rate of Human Immunodeficiency Virus Type 1 than That Predicted from the Fidelity of Purified Reverse Transcriptase

The level of genetic variation of human immunodeficiency virus type 1 (HIV-1) is high relative to that of some other retroviruses. Purified HIV-1 reverse transcriptase has high error rates in cell-free systems, which suggests an explanation for high genetic variation. Mansky and Temin (L. M. Mansky and H. M. Temin, J Virol 69:5087-5094, 1995, http://jvi.asm.org/content/69/8/5087.abstract) developed an in vivo assay for detecting mutations in an HIV-1 vector containing the lacZ gene as a reporter for mutations, which allowed quantification of the mutations occurring during a single round of HIV-1 replication. The most commonly detected mutations were base substitution mutations, G-to-A and C-to-T transition mutations, and frameshift mutations in runs of T's and A's. The in vivo mutation rates for HIV-1 were three to seven times higher than those reported for two other retroviruses. However, in vivo mutation rates were about 20 -fold lower than the error rate of purified HIV-1 reverse transcriptase with the same target sequence. This finding indicates that HIV-1 reverse transcription in vivo is not as error prone as predicted from the fidelity of purified reverse transcriptase in cell-free studies, suggesting that the fidelity of purified HIV-1 reverse transcriptase may not accurately reflect the level of genetic variation in a natural infection.

\section{High-Level Hepatitis B Virus Replication in Transgenic Mice}

Hepatitis B virus (HBV) can cause acute and chronic hepatitis, cirrhosis, and hepatocellular carcinoma. Because of the limited host range of HBV and the inability to propagate it in cell culture, other members of the hepadnavirus family have been studied, including woodchuck hepatitis virus and duck hepatitis B virus, which infect their native species. Importantly, duck hepatitis B virus can replicate in tissue culture. Guidotti et al. (L. G. Guidotti, B. Matzke, H. Schaller, and F. V. Chisari, J Virol 69:61586169, 1995, http://jvi.asm.org/content/69/10/6158.abstract) produced HBV transgenic mice whose hepatocytes replicate the virus at levels comparable to that in the infected livers of patients with chronic hepatitis without any evidence of cytopathology. Highlevel viral gene expression was obtained in liver and kidney tissues of three independent lineages. A terminally redundant viral DNA construct (HBV 1.3) was used that starts upstream of the HBV enhancer and extends completely around the circular viral genome, ending downstream of the polyadenylation site in HBV. While other investigators had produced transgenic mice capable of replicating HBV, virus replication occurred at a low level in these animals. The new transgenic lineages produced in this report allowed the investigation of the influence of viral and host factors on HBV replication and pathogenesis, including the immune response, to control HBV replication in a genetically defined small-animal system. 